\title{
MAGIC OBSERVATIONS OF VERY HIGH ENERGY $\gamma$-RAYS FROM HESS J1813-178
}

J. Albert, ${ }^{1}$ E. Aliu,${ }^{2}$ H. Anderhub,${ }^{3}$ P. Antoranz,${ }^{4}$ A. Armada,${ }^{2}$ M. Asensio,${ }^{4}$ C. Baixeras,${ }^{5}$ J. A. Barrio, ${ }^{4}$ M. Bartel,${ }^{6}$ H. Bartko, ${ }^{7}$ D. Bastieri,${ }^{8}$ R. Bavikadi, ${ }^{9}$ W. Bednarek,${ }^{10}$ K. Berger,${ }^{1}$ C. Bigongiari,${ }^{8}$ A. Biland,${ }^{3}$ E. Bisesi,${ }^{9}$ O. Blanch, ${ }^{2}$ R. K. Bock, ${ }^{7}$ T. Bretz, ${ }^{1}$ I. Britvitch, ${ }^{3}$ M. Camara,${ }^{4}$ A. Chilingarian,${ }^{11}$ S. Ciprini,${ }^{12}$ J. A. Coarasa,${ }^{7}$ S. Commichau, ${ }^{3}$ J. L. Contreras, ${ }^{4}$ J. Cortina, ${ }^{2}$ V. Curtev, ${ }^{6}$ V. Danielyan, ${ }^{11}$ F. Dazzi,${ }^{8}$ A. De Angelis, ${ }^{9}$ R. de los Reyes, ${ }^{4}$ B. De Lotto, ${ }^{9}$ E. Domingo-Santamaria,${ }^{2}$ D. Dorner,${ }^{1}$ M. Doro,${ }^{8}$ M. Errando, ${ }^{2}$ M. Fagiolini,${ }^{13}$ D. Ference, ${ }^{14}$ E. Fernández,${ }^{2}$ R. Firpo, ${ }^{2}$ J. Flix, ${ }^{2}$ M. V. Fonseca, ${ }^{4}$ L. Font,${ }^{5}$ N. Galante,${ }^{13}$ M. GarczarczyK, ${ }^{7}$ M. Gaug,${ }^{2}$ J. Gebauer,${ }^{7}$ M. Giller, ${ }^{10}$ F. Goebel, ${ }^{7}$ D. Hakobyan, ${ }^{11}$ M. Hayashida, ${ }^{7}$ T. Hengstebeck,${ }^{15}$ D. Höhne, ${ }^{1}$ J. Hose,${ }^{7}$ P. Jacon, ${ }^{10}$ O. Kalekin,,${ }^{15}$ D. Kranich, ${ }^{14}$ A. Laille,${ }^{14}$ T. Lenisa, ${ }^{9}$ P. Liebing,${ }^{7}$ E. Lindfors,${ }^{12}$ F. Longo,${ }^{9}$ M. López,${ }^{4}$ J. LópeZ,${ }^{2}$ E. Lorenz,${ }^{3,7}$ F. Lucarelli,${ }^{4}$ P. Majumdar, ${ }^{7}$ G. Maneva, ${ }^{16}$ K. Mannheim, ${ }^{1}$ M. Mariotti,${ }^{8}$ M. Martínez,${ }^{2}$ K. Mase, ${ }^{7}$ D. Mazin,,${ }^{7}$ C. Merck,${ }^{7}$ M. Merck,${ }^{1}$ M. Meucci,${ }^{13}$ M. Meyer, ${ }^{1}$ J. M. Miranda, ${ }^{4}$ R. Mirzoyan,${ }^{7}$ S. Mizobuchi, ${ }^{7}$ A. Moralejo,,${ }^{8}$ K. Nilsson, ${ }^{12}$ E. Oña-Wilhelmi, ${ }^{2}$ R. Orduña,${ }^{5}$ N. Otte,${ }^{7}$ I. Oya,${ }^{4}$ D. Paneque, ${ }^{7}$ R. Paoletti,${ }^{13}$ M. Pasanen, ${ }^{12}$ D. Pascoli, ${ }^{8}$ F. Pauss, ${ }^{3}$ N. Pavel,${ }^{15}$ R. Pegna,${ }^{13}$ L. Peruzzo,${ }^{8}$ A. Piccioli,${ }^{13}$ E. Prandini,${ }^{8}$ J. Rico, ${ }^{2}$ W. Rhode, ${ }^{6}$ B. Riegel, ${ }^{1}$ M. Rissi, ${ }^{3}$ A. Robert,${ }^{5}$ G. Rossato, ${ }^{8}$ S. Rügamer, ${ }^{1}$ A. Saggion,${ }^{8}$ A. Sanchez,${ }^{8}$ P. Sartori,${ }^{8}$ V. Scalzotto, ${ }^{8}$ R. Schmitt,${ }^{1}$ T. Schweizer, ${ }^{15}$ M. Shayduk, ${ }^{15}$ K. Shinozaki ${ }^{7}$ S. Shore,${ }^{13}$ N. Sidro, ${ }^{2}$ A. Sillanpää, ${ }^{12}$ D. Sobczynska, ${ }^{10}$ A. Stamerra, ${ }^{13}$ L. Stark, ${ }^{3}$ L. Takalo,,${ }^{12}$ P. Temnikov, ${ }^{16}$ D. Tescaro, ${ }^{8}$ M. Teshima, ${ }^{7}$ N. Tonello, ${ }^{7}$ A. Torres,${ }^{5}$ D. F. Torres, ${ }^{2}$ N. Turini,${ }^{13}$ H. Vankov, ${ }^{16}$ V. Vitale, ${ }^{9}$ R. Wagner, ${ }^{7}$ T. Wibig, ${ }^{10}$ W. Wittek, ${ }^{7}$ and J. Zapatero ${ }^{5}$ Received 2005 October 17; accepted 2005 December 9; published 2006 January 10

\section{ABSTRACT}

Recently, the HESS collaboration has reported the detection of $\gamma$-ray emission above a few hundred $\mathrm{GeV}$ from eight new sources located close to the Galactic plane. The source HESS J1813-178 has sparked particular interest, as subsequent radio observations imply an association with supernova remnant G12.82-0.02. Triggered by the detection in very high energy $\gamma$-rays, a positionally coincident source has also been found in INTEGRAL and ASCA data. In this Letter we present MAGIC observations of HESS J1813-178, resulting in the detection of a differential $\gamma$-ray flux consistent with a hard-slope power law, described as $d N_{\gamma} /(d A d t d E)=(3.3 \pm$

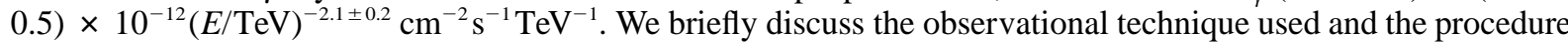
implemented for the data analysis, and we put this detection in the perspective of multifrequency observations.

Subject headings: acceleration of particles — gamma rays: observations — supernova remnants

\section{INTRODUCTION}

In the Galactic plane scan performed with the HESS (High Energy Stereoscopic System) Cerenkov array in 2004, with a flux sensitivity of $3 \%$ crab units for $\gamma$-rays above $100 \mathrm{GeV}$, eight sources were discovered (Aharonian et al. 2005, 2006). One of the newly detected $\gamma$-ray sources was HESS J1813178. At the beginning, HESS J1813-178 could not be identified and was assumed to be a "dark particle accelerator," without reported counterparts at lower frequencies.

Since the original discovery, HESS J1813-178 has been associated with the supernova remnant (SNR) G12.82-0.02

\footnotetext{
${ }^{1}$ Universität Würzburg, D-97074 Würzburg, Germany.

${ }^{2}$ Institut de Fisica d'Altes Energies, Edifici Cn., E-08193 Bellaterra (Barcelona), Spain.

${ }^{3}$ ETH Hönggerberg, CH-8093 Zürich, Switzerland.

${ }^{4}$ Universidad Complutense, E-28040 Madrid, Spain

${ }^{5}$ Universitat Autonoma de Barcelona, E-08193 Bellaterra, Spain.

${ }^{6}$ Universität Dortmund, D-44227 Dortmund, Germany.

${ }^{7}$ Max-Planck-Institut für Physik, D-80805 München, Germany; correspondence should be addressed to H. Bartko, hbartko@mppmu.mpg.de.

${ }^{8}$ Università di Padova and INFN, I-35131 Padua, Italy.

${ }^{9}$ Università di Udine and INFN Trieste, I-33100 Udine, Italy.

${ }^{10}$ University of Lodz, PL-90236 Lodz, Poland.

${ }^{11}$ Yerevan Physics Institute, AM-375036 Yerevan, Armenia.

${ }^{12}$ Tuorla Observatory, FI-21500 Piikkiö, Finland.

${ }^{13}$ Università di Siena and INFN Pisa, I-53100 Siena, Italy.

${ }^{14}$ University of California, Davis, CA 95616-8677.

${ }^{15}$ Humboldt-Universität zu Berlin, D-12489 Berlin, Germany.

${ }^{16}$ Institute for Nuclear Research and Nuclear Energy, BG-1784 Sofia, Bulgaria.
}

(Ubertini et al. 2005; Brogan et al. 2005; Helfand et al. 2005). One may still not exclude this coincidence as being the result of just a chance association. Aharonian et al. (2005) state a probability of $6 \%$ that one of their new sources is by chance spatially consistent with an SNR. Nevertheless, the properties of SNR G12.82-0.02, the multifrequency spectral energy distribution (SED), and the flux and spectrum of the high-energy $\gamma$-rays detected from this direction appear to be consistent with an SNR origin.

HESS J1813-178 has been found to be nearly pointlike $\left(\sigma_{\text {source }}=2 \cdot 2\right)$ by Aharonian et al. (2006). Given the size of the SNR, the angular resolution of the HESS telescope, and the depth of the observations, the source size does not rule out a possible shell origin. The $\gamma$-ray source lies at $10^{\prime}$ from the center of the radio source W33. This patch of the sky is highly obscured and has every indication of being a recent star formation region (Churchwell 1990).

In this Letter we present MAGIC (Major Atmospheric Gamma Imaging Cerenkov telescope) observations of HESS J1813-178. We briefly discuss the observational technique used and the procedure implemented for the data analysis. We derive a high-energy $\gamma$-ray spectrum of the source and analyze it in the perspective of multifrequency observations.

\section{OBSERVATIONS}

MAGIC (see, e.g., Baixeras et al. 2004 and Cortina et al. 2005) is currently the largest single-dish Imaging Air Cerenkov Telescope 
(IACT) in operation. Located on the Canary Island La Palma (N28.8, W17.8, $2200 \mathrm{~m}$ above sea level), the telescope has a $17 \mathrm{~m}$ diameter tessellated parabolic mirror, supported by a lightweight carbon-fiber frame. It is equipped with a high-efficiency 576 pixel 3.5 field-of-view photomultiplier camera. The analog signals are transported via optical fibers to the trigger electronics and are read out by a 300 MSamples $\mathrm{s}^{-1}$ FADC system.

At La Palma, HESS J1813-178 culminates at about $47^{\circ}$ zenith angle (ZA). The large ZA implies a high-energy threshold of about $400 \mathrm{GeV}$ for MAGIC observations. It also provides a large effective collection area (see, e.g., Konopelko et al. 1999). The sky region around the location of HESS J1813-178 has a relatively high and nonuniform level of light. Within a distance of $1^{\circ}$ from HESS J1813-178, there are no stars brighter than $8 \mathrm{mag}$, with the star field being brighter in the region southwest of the source.

The MAGIC observations were carried out in the falsesource tracking (wobble) mode (Fomin et al. 1994). The sky directions (W1, W2) to be tracked are chosen such that in the camera, the sky field relative to the source position is similar to the sky field relative to the mirror source position (antisource position). The source direction is in both cases 0.4 offset from the camera center. In Figure 1 these two tracking positions are shown as white stars. During wobble mode data taking, 50\% of the data is taken at $\mathrm{W} 1$, and $50 \%$ at W2, switching (wobbling) between the two directions every 30 minutes. This observation mode allowed us to make a reliable background estimation that is least affected by the large ZA and inhomogeneous star field.

\section{DATA ANALYSIS}

HESS J1813-178 was observed for a total of $25 \mathrm{hr}$ in the period 2005 June-July $\left(\mathrm{ZA} \leq 52^{\circ}\right)$. About 15 million triggers have been recorded. The calibration of the raw data of the MAGIC camera is explained in Gaug et al. (2005). Image cleaning tail cuts were applied: Pixels are only considered to be part of the image if their reconstructed charge signal is larger than 10 photoelectrons (core pixels) or if their charge is larger than 5 photoelectrons and they have at least one neighboring core pixel. These tail cuts are accordingly scaled for the larger outer pixels of the MAGIC camera. The camera images are characterized by image parameters (Hillas 1985). After the image cleaning and rejection of broken runs, about 10 million events remained for further analysis. These data were processed for $\gamma /$ hadron separation, in a similar way as described in Fegan (1997).

In this analysis, the Random Forest (RF) method (see Bock et al. 2004 for a detailed description) was applied for the $\gamma /$ hadron separation and the energy estimation. For the training of the RF, a sample of Monte Carlo-generated $\gamma$-ray showers was used to represent the $\gamma$-rays, and a randomly chosen subset of the measured data was used to represent the background. The Monte Carlo (MC) $\gamma$-ray showers were generated between ZA $=47^{\circ}$ and $54^{\circ}$, with energies between $10 \mathrm{GeV}$ and $30 \mathrm{TeV}$, respectively. The spectral index of the generated differential spectrum $d N_{\gamma} / d E \sim E^{\Gamma}$ was chosen as $\Gamma=-2.6$, in agreement with the MAGIC-observed energy spectrum of the Crab Nebula (Wagner et al. 2005). ${ }^{17}$ The source-position-independent image parameters SIZE, WIDTH, LENGTH, CONC (Hillas 1985), and the third moment along the major image axis, as well as the

\footnotetext{
${ }^{17}$ In order to develop and verify the analysis at high zenith angles, Crab data in the interesting ZA range around $50^{\circ}$ were taken in 2005 January. From that sample, we determined the Crab energy spectrum and found it to be consistent with other existing measurements (see Fig. 3, upper curve).
}

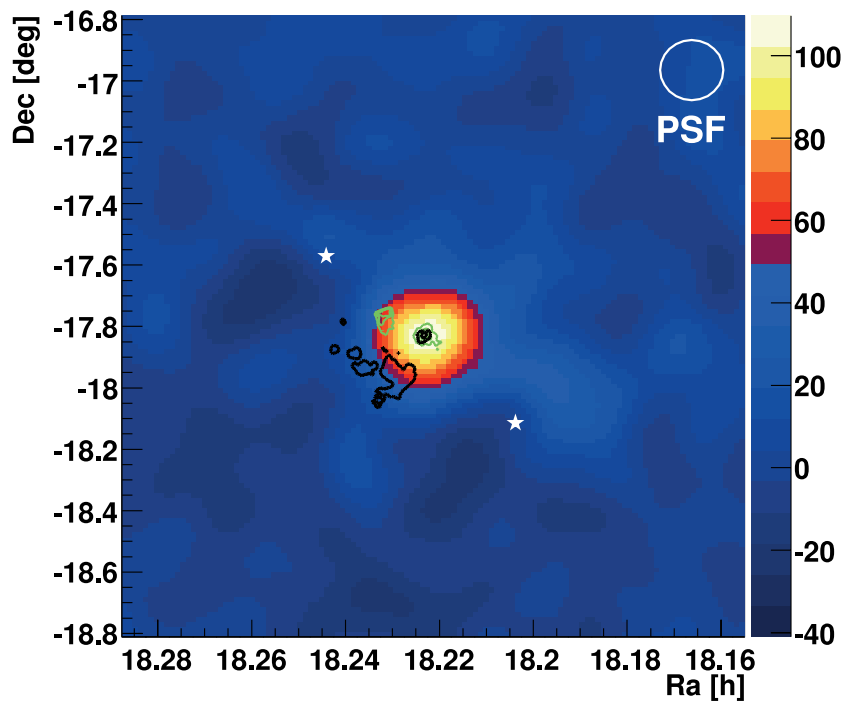

FIG. 1.-Sky map of $\gamma$-ray candidate events (background-subtracted) in the direction of HESS J1813-178 for SIZE $\geq 600$ photoelectrons (corresponding to an energy threshold of about $1 \mathrm{TeV}$ ). Overlaid are contours of $90 \mathrm{~cm}$ VLA radio (black) and ASCA X-ray data (green) from Brogan et al. (2005). The two white stars denote the tracking positions W1, W2 in the wobble mode.

source-position-dependent parameter DIST (Hillas 1985), were selected to parameterize the shower images. After the training, the RF method allows us to calculate for every event a parameter, dubbed "hadronness," which is a measure of the probability that the event belongs to the background. The $\gamma$-ray sample is defined by selecting showers with a hadronness below a specified value. An independent sample of MC $\gamma$-ray showers was used to determine the efficiency of the applied cuts.

For each event, its original sky position is determined by using the DISP method (Fomin et al. 1994; Lessard et al. 2001). At this stage, only source-independent image parameters are used in the RF training. Figure 1 shows the sky map of $\gamma$-ray candidate events (background-subtracted) from the direction of HESS J1813-178. It is smoothed with a two-dimensional Gaussian with a standard deviation of 0.1 . To provide a good angular resolution, a tight hadronness cut and a lower SIZE cut of 600 photoelectrons have been applied. The SIZE cut corresponds to an energy threshold of about $1 \mathrm{TeV}$. The sky map is overlaid with contours of $90 \mathrm{~cm}$ VLA radio and $A S C A$ $\mathrm{X}$-ray data from Brogan et al. (2005). The excess is centered at (R.A., decl. $)=\left(18^{\mathrm{h}} 13^{\mathrm{m}} 27^{\mathrm{s}},-17^{\circ} 48^{\prime} 40^{\prime \prime}\right)$ and coincides well with the position of SNR G12.82-0.02. The systematic pointing uncertainty is estimated to be $2^{\prime}$ (for a description of the MAGIC telescope drive system, see Bretz et al. 2003) and might in future be greatly reduced with the MAGIC star-field monitor (Riegel et al. 2005). Apart from the main excess coincident with HESS J1813-178, there are no other significant excesses present.

Figure 2 shows the distribution of the squared angular distance, $\theta^{2}$, between the reconstructed shower direction and the nominal object position. The observed excess in the direction of HESS J1813-178 has a significance of $10.6 \sigma$ (according to eq. [17] of Li \& Ma 1983). Within errors, the source position and the flux level are compatible with the measurement of HESS (Aharonian et al. 2006).

For the determination of the energy spectrum, the RF was trained, including the source-dependent image parameter DIST, with respect to the nominal excess position. A loose cut on the 


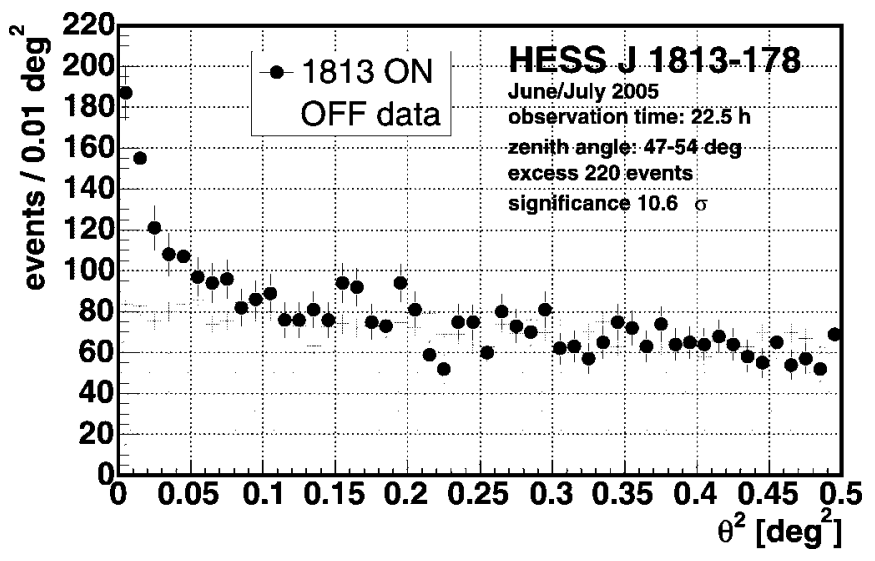

FIG. 2.-Distributions of $\theta^{2}$-values for the source and antisource (see text) for SIZE $\geq 600$ photoelectrons (corresponding to an energy threshold of about $1 \mathrm{TeV})$.

hadronness was used. Above the low-energy turnon, the cut efficiency reaches about $70 \%$, corresponding to an effective collection area for $\gamma$-ray showers of about $180,000 \mathrm{~m}^{2}$. Figure 3 shows the reconstructed very high energy (VHE) $\gamma$-ray spectrum of HESS J1813-178 after the unfolding with the instrumental energy resolution. The measured spectral points are fit by a simple power-law spectrum, taking the full instrumental energy resolution into account as described in Mizobuchi et al. (2005). The result is given by $\left(\chi^{2} / \mathrm{dof}=5.3 / 5\right)$ :

$$
\begin{aligned}
\frac{d N_{\gamma}}{d A d t d E}= & (3.3 \pm 0.5) \times 10^{-12} \\
& \times(E / \mathrm{TeV})^{-2.1 \pm 0.2} \mathrm{~cm}^{-2} \mathrm{~s}^{-1} \mathrm{TeV}^{-1}
\end{aligned}
$$

The quoted errors $\left(\begin{array}{ll}1 & \sigma\end{array}\right)$ are purely statistical. The systematic error is estimated to be $35 \%$ in the flux level determination and 0.2 in the spectral index. Within errors, the flux is steady in the timescales explored within these observations (weeks) as well as in the year-long time span between the MAGIC and HESS pointings.

\section{DISCUSSION}

Shortly after the discovery of HESS J1813-178, X-ray emission was found in ASCA data coming from the source AX J1813-178 (aka AGPS 273.4-17.8; Brogan et al. 2005; see also Ubertini et al. 2005). X-ray emission detected by $A S C A$ is predominantly nonthermal and is compatible with that expected from a pulsar wind nebula or an SNR shell. X-raypulsed emission has not been detected, but the quality and amount of the data do not imply strong constraints (Brogan et al. 2005). Statistically, X-ray data are not good enough to unambiguously separate a pure power-law contribution from a power-law + thermal contribution either. However, in both cases, none of the two-component fits yield a significantly different absorbing column density or photon index when compared with a single power-law fit (Brogan et al. 2005). Data analysis from the INTEGRAL satellite also showed a soft and luminous source at the same location, in the $20-100 \mathrm{keV}$ range (Ubertini et al. 2005).

Simultaneously with this X-ray match, HESS J1813-178 was also found as a nonthermal source in radio data, using observations with VLA (90 and $20 \mathrm{~cm})$, Bonn $(3 \mathrm{~cm})$, Parkes $(6 \mathrm{~cm})$, and Nobeyama $(3 \mathrm{~cm})$ telescopes (Brogan et al. 2005;

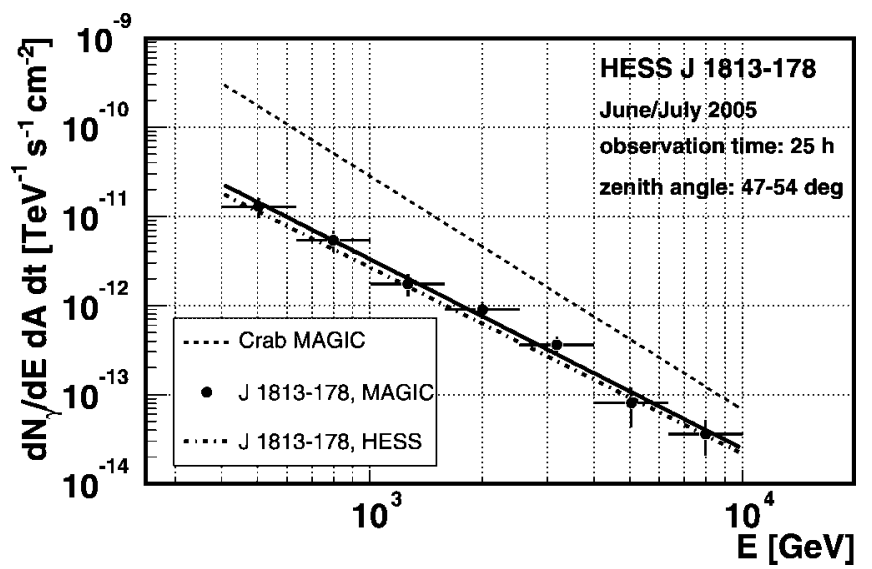

FIG. 3.-Reconstructed VHE $\gamma$-ray spectrum of HESS J1813-178. The spectral index is $-2.1 \pm 0.2$, and the integral flux above $400 \mathrm{GeV}$ is about $8 \%$ of the Crab Nebula (statistical errors only). The dashed line shows the spectrum of the Crab Nebula as measured by MAGIC (Wagner et al. 2005). The dot-dashed line shows the results of the HESS collaboration (Aharonian et al. 2006).

Helfand et al. 2005). These groups discovered a shell-type supernova remnant (SNR G12.8-0.0) with a section of the shell coinciding with HESS J1813-178. The radio spectral index was found to be $-0.48 \pm 0.03$. There are no known radio pulsars detected at the HESS J1813-178 position (Manchester et al. 2005).

Brogan et al. (2005) conclude that SNR G12.8-0.0 should lie at or beyond the distance of $\mathrm{W} 33, \sim 4 \mathrm{kpc}$. They have derived a high column density of $N_{\mathrm{H}} \sim 10^{23} \mathrm{~cm}^{-2}$ from the ASCA data that suggests a significant source of absorption in the foreground.

The multiwavelength emission coming from the direction of HESS J1813-178 is shown in Figure 4, including the new MAGIC data at high energies. We have compared hadronic (neutral pion decay) and leptonic (inverse Compton) emission models with the high-energy $\gamma$-ray data (for a review, see Torres et al. 2003). In the case of hadronic models, the observed $\gamma$ ray luminosity $\left(2.5 \times 10^{34} \mathrm{ergs} \mathrm{s}^{-1}\right.$ between 0.4 and $6 \mathrm{TeV}$, at $4 \mathrm{kpc}$ ) implies that the required density of matter is $\sim 6 \mathrm{~cm}^{-3}$. The $\gamma$-ray production region is presumed to be the whole SNR

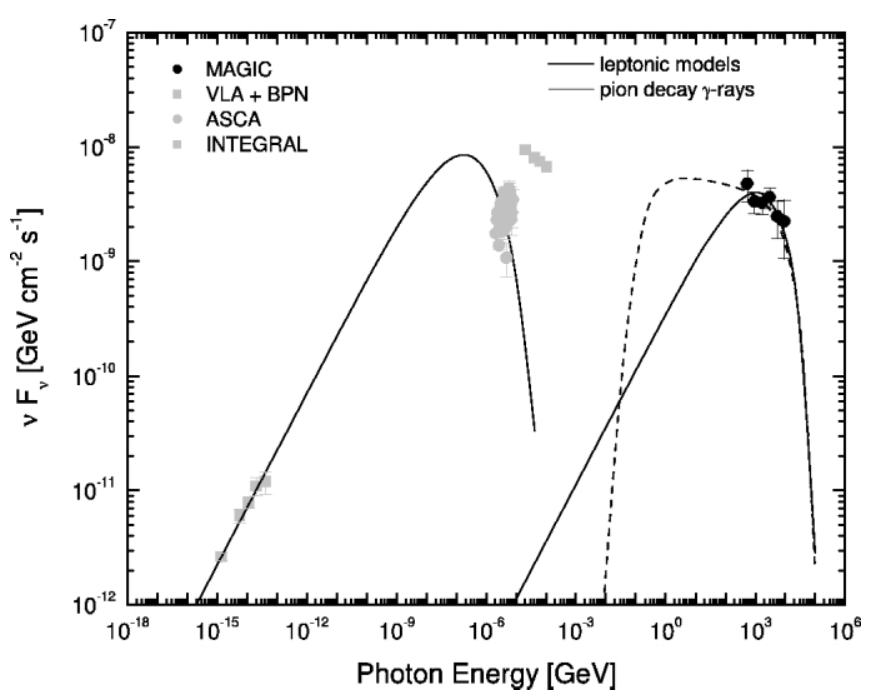

FIG. 4.-Leptonic and hadronic models for the J1813-178 data. Details are given in the text. Radio data are from the VLA, Bonn, Parkes, and Nobeyama observatories (Brogan et al. 2005); X-ray and hard X-ray data are from ASCA and INTEGRAL (Ubertini et al. 2005; Brogan et al. 2005). 
volume. An acceleration efficiency of hadrons of the order of $3 \%$ and a supernova power of $10^{51} \mathrm{ergs}$ were assumed. Therefore, relativistic hadrons need only about $2 M_{\odot}$ of target mass inside the SNR volume ( $1.5 \mathrm{pc}$ radius at $4 \mathrm{kpc}$ distance $)$ in order to be consistent with the observed luminosity. Alternatively, the target mass for the cosmic-ray spectrum can be provided by a small cloud of a few solar masses located in a region close to the SNR shell. For the model shown in Figure 4, we have assumed that the proton distribution is described by $d N_{p} /(d V d E)=A_{p}(E / \mathrm{GeV})^{-\alpha} \exp \left(-E / E_{\max }\right) \quad \mathrm{GeV}^{-1} \quad \mathrm{~cm}^{-3}$, where $A_{p}$ is a dimensionless normalization constant. We found that $\alpha=2.1$ and $E_{\max }=100 \mathrm{TeV}$ provide a good fit to the data, and that the normalization constant is such that the amount of supernova explosion energy converted into relativistic cosmic rays need not to be more than a few percent to agree with MAGIC observations.

For leptonic models, we have assumed a similarly described distribution of relativistic electrons $d N_{e} /(d V d E)=$ $A_{e}(E / \mathrm{GeV})^{-\alpha_{e}} \exp \left(-E / E_{\max , e}\right) \mathrm{GeV}^{-1} \mathrm{~cm}^{-3}$. We found that several different inverse Compton models produce reasonably good fits at high energies, e.g., $\alpha_{e}=2.0$ and $E_{\max , e}=20 \mathrm{TeV}$, and $\alpha_{e}=2.1$ and 2.2 and $E_{\max , e}=30 \mathrm{TeV}$, all having their energetics at ease with the energy assumed to be released by the supernova explosion. The source of target photons for these models was assumed to be the cosmic microwave background. The radio spectrum at lower energies is fit best by a slope of $\alpha_{e}=2.0$. It is computed as synchrotron emission of the same electron population. In the model shown in Figure 4, a magnetic filling fraction of about $20 \%$, a magnetic field of $10 \mu \mathrm{G}, E_{\max , e}=20 \mathrm{TeV}$, and $\alpha_{e}=2.0$ have been adopted. This model is similar to one of the models presented by Brogan et al. (2005; the blue line in their Fig. 3; $E_{\max , e}=30, \alpha_{e}=2.0$ ), without yet having a high-energy $\gamma$-ray spectrum. The soft X-ray data (ASCA) plotted correspond to those shown by Brogan et al. (2005; the black data points in their Fig. 3). Our model as well as the model by Brogan et al. are in rough agreement with the MAGIC data, with radio data, and also roughly with the X-ray data, even if the spectral behavior in the $A S C A$ energy range (the slope) is somewhat different. That is, we have priviledged the radio data in fitting this SED as an example; a better X-ray fitting could be obtained at the expense of worse radio data. It is worth noticing that the hard X-ray data of INTEGRAL (Ubertini et al. 2005) cannot be fit well with the same electron population that is assumed to produce higher and lower energy photons. Nevertheless, as one can see in Figure 2 of Ubertini et al. (2005), the $A S C A$, MAGIC, and HESS sources match well spatially, while the INTEGRAL/IBIS source is spatially only marginally compatible with them. Further multifrequency observations in the X-ray and hard X-ray regime are needed to obtain definite conclusions as to what level the leptonic models need two electron populations.

\section{CONCLUDING REMARKS}

The detection of HESS J1813-178 using the MAGIC telescope confirms a new VHE $\gamma$-ray source in the Galactic plane. A reasonably large data set was collected from observations at large zenith angles to infer the spectrum of this source up to energies of about $10 \mathrm{TeV}$. Between $400 \mathrm{GeV}$ and $10 \mathrm{TeV}$, the differential energy spectrum can be fit with a power law of slope $\Gamma=-2.1 \pm 0.2$. These data can be used to cross-calibrate HESS and MAGIC; their independent observations show satisfactory agreement.

Multifrequency data in the radio, X-ray, and $\gamma$-ray band imply a connection between HESS J1813-178 and SNR G12.82-0.02 (Helfand et al. 2005; Ubertini et al. 2005; Brogan et al. 2005). Generally, hard $\gamma$-ray spectra are expected from SNRs due to the Fermi acceleration of cosmic rays (Ginzburg \& Syrovatskii 1964; Torres et al. 2003). The hard spectrum determined for HESS J1813-178 may be a further hint of its association with the SNR G12.82-0.02.

Present data are not sufficient to discriminate between existing models for different acceleration mechanisms. Future observations at lower energies with improved gamma-ray telescopes and/or the Gamma-Ray Large Area Space Telescope (GLAST) will undoubtedly shed more light on the existing leptonic and hadronic models. Decisive information concerning hadronic acceleration mechanisms is also likely to come from future neutrino telescopes like IceCube (Ahrens et al. 2004).

We would like to thank the IAC for the excellent working conditions at the Observatory de los Muchachos in La Palma. The support of the German BMBF and MPG, the Italian INFN, and the Spanish CICYT is gratefully acknowledged. This work was also supported by ETH research grant TH 34/04 3 and the Polish MNiI grant 1P03D01028.

\section{REFERENCES}

Aharonian, F., et al. 2005, Science, 307, 1938 2006, ApJ, 636, 777

Ahrens, J., et al. 2004, Astropart. Phys., 20, 507

Baixeras, C., et al. 2004, Nucl. Instrum. Methods Phys. Res. A, 518, 188

Bock, R. K., et al. 2004, Nucl. Instrum. Methods Phys. Res. A, 516, 511

Bretz, T., et al. 2003, Proc. 28th Int. Cosmic Ray Conf. (Tsukuba), 2943

Brogan, C. L., Gaensler, B. M., Gelfand, J. D., Lazendic, J. S., Lazio, T. J. W., Kassim, N. E., \& McClure-Griffiths, N. M. 2005, ApJ, 629, L105

Churchwell, E. 1990, A\&A Rev., 2, 79

Cortina, J., et al. 2005, Proc. 29th Int. Cosmic Ray Conf. (Pune), in press (astro-ph/0508274)

Fegan, D. J. 1997, J. Phys. G, 23, 1013

Fomin, V. P., Stepanian, A. A., Lamb, R. C., Lewis, D. A., Punch, M., \& Weekes, T. C. 1994, Astropart. Phys., 2, 137

Gaug, M., et al. 2005, Proc. 29th Int. Cosmic Ray Conf. (Pune), in press (astro$\mathrm{ph} / 0508274)$

Ginzburg, V. L., \& Syrovatskii, S. I. 1964, The Origin of Cosmic Rays (New York: Macmillan)
Helfand, D. J., Becker, R. H., \& White, R. L. 2005, ApJL, submitted (astro$\mathrm{ph} / 0505392)$

Hillas, A. M. 1985, Proc. 19th Int. Cosmic Ray Conf. (La Jolla), 3, 445

Konopelko, A., Aharonian, F., Hemberger, M., Hofmann, W., Kettler, J., Pühlhofer, G., \& Völk, H. J. 1999, J. Phys. G, 25, 1989

Lessard, R. W., Buckley, J. H., Connaughton, V., \& Le Bohec, S. 2001, Astropart. Phys., 15, 1

Li, T.-P., \& Ma, Y.-Q. 1983, ApJ, 272, 317

Manchester, R. N., Hobbs, G. B., Teoh, A., \& Hobbs, M. 2005, AJ, 129, 1993

Mizobuchi, S., et al. 2005, Proc. 29th Int. Cosmic Ray Conf. (Pune), in press (astro-ph/0508274)

Riegel, B., et al. 2005, Proc. 29th Int. Cosmic Ray Conf. (Pune), in press (astro-ph/0508274)

Torres, D. F., Romero, G. E., Dame, T. M., Combi, J. A., \& Butt, Y. M. 2003, Phys. Rep., 382, 303

Ubertini, P., et al. 2005, ApJ, 629, L109

Wagner, R., et al. 2005, Proc. 29th Int. Cosmic Ray Conf. (Pune), in press (astro-ph/0508244) 